\title{
P02-6-6 Poster session
}

\section{Role of endogenous PGE2 in liver repair after hepatic ischemia/reperfusion}

\author{
Yoshiya Ito ${ }^{1}$, Nobuyuki Nishizawa ${ }^{1,2}$, Koji Eshima ${ }^{3}$, Hirotoki Ohkubo ${ }^{4}$, Tomoyoshi Inoue ${ }^{1}$, \\ Hideki Amano ${ }^{1}$, Masataka Majima ${ }^{1}$
}

${ }^{I}$ Pharmacology, Kitasato University School of Medicine, Japan, ${ }^{2}$ Surgery, Kitasato University School of Medicine, Japan, Immunology, Kitasato University School of Medicine, Japan, ${ }^{4}$ Cardiiovascular Surgery, Kitasato University School of Medicine, Japan

Background: Hepatic ischemia/reperfusion (I/R) injury is a major adverse reaction to liver surgery. Impaired liver repair following hepatic $\mathrm{I} / \mathrm{R}$ affects the outcome of the patients. This study aims to examine the role of endogenous PGE2 produced by inducible microsomal PGE synthase-1 (mPGES-1), a terminal enzyme of PGE2 generation, in liver repair following hepatic I/R.

Methods: mPGES-1 deficient (mPGES-1-/-) mice or their wild counterparts (WT) were subjected to partial hepatic ischemia followed by reperfusion. ALT, necrotic area, cytokines, and cellular growth factors were determined and compared. The hepatic macrophages were assessed by immunofluorescence and flow cytometry analysis.

Results: Compared with WT mice, mPGES-1-/- mice exhibited reductions in ALT, necrotic area, and enhancement of growth factors (HGF and EGF) at $48 \mathrm{~h}$ and $96 \mathrm{~h}$ post-reperfusion (repair phase). mPGES-1-/- mice also showed increases in hepatic macrophage accumulation into the injured region, with down-regulated expression of genes related to proinflammatory macrophage phenotype and up-regulated genes related to restorative macrophage phenotype. Flow cytometry analysis revealed reduction in Ly6Chigh macrophages together with pro-inflammatory mediators, and increasing Ly6Clow macrophages with anti-inflammatory mediators in mPGES-1-/- mice. In vitro, mPGES-1 was expressed in cultured bone marrow (BM)-derived macrophages polarized toward the pro-inflammatory, but not the restorative profile. Adoptive transfer of BM-derived mPGES-1-deficient macrophages into WT mice promoted liver repair. Mice treated with the mPGES-1 inhibitor Compound III displayed acceleration of liver repair.

Conclusions: These results suggest that mPGES-1 in pro-inflammatory macrophages impairs liver repair after hepatic I/R injury. Targeting mPGES-1 in liver repair following hepatic I/R could be of therapeutic benefit. 